\title{
ÉTUDE ASYMPTOTIQUE D'UNE MARCHE ALÉATOIRE CENTRIFUGE
}

\author{
JEAN-DENIS FOUKS, EMMANUEL LESIGNE ET MARC PEIGNÉ
}

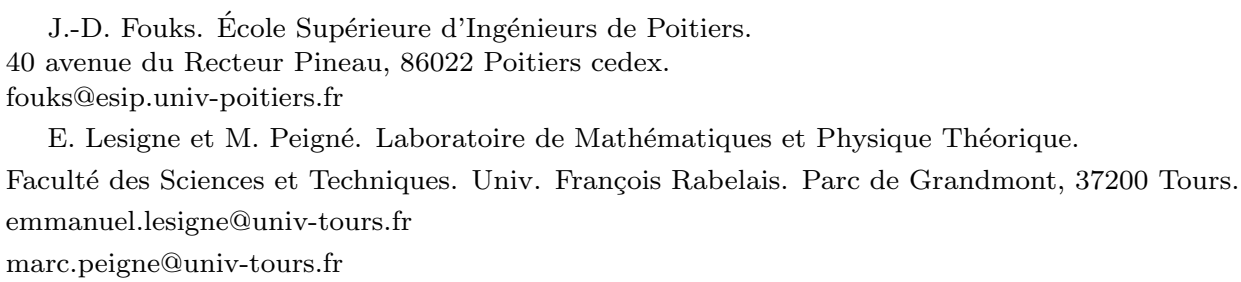

RÉSumÉ. La marche aléatoire centrifuge est une chaîne de Markov dans l'espace euclidien dont les transitions sont celles d'une marche aléatoire symérique ordinaire perturbées par une dérive centrifuge. Nous étudions le comportement de ce processus en moyenne, par trajectoires individuelles et en loi quand le temps tend vers l'infini.

\section{SOMMAIRE}

Introduction $\quad 2$

Présentation de la chaîne étudiée, sur un exemple simple. $\quad 2$

Méthodes et résultats 3

Motivation extra-mathématique $\quad 3$

1. La marche centrifuge 3

1.1. Présentation du modèle 3

1.2. Quelques notations 5

1.3. Hypothèses de non-dégénérescence 5

1.4. Principaux résultats 5

2. Comportement en norme 6

2.1. Estimations de moments 6

2.2. Loi des grands nombres 9

2.3. Théorème limite central 10

3. Comportement en direction 12

3.1. Convergence 12

3.2. Piégeage dans les cônes et support de la loi limite 14

4. Indépendance asymptotique de la norme et de la direction 18

5. Questions 20

5.1. Loi limite de la direction 21

5.2. Théorème limite local 22

6. Appendice : TLC pour martingales avec paramètre 23

$\begin{array}{ll}\text { References } & 25\end{array}$

Date: novembre 2004. 


\section{INTRODUCTION}

L'objet de cet article est l'étude du comportement en temps grand d'une marche aléatoire dans l'espace euclidien, soumise à une dérive centrifuge. L'étude précise de cette chaîne de Markov est justifiée par une double motivation. D'une part la question est apparue en dehors du champ usuel du Calcul des Probabilités, lui ôtant ainsi le risque de généralisation purement gratuite, d'autre part elle conduit à des résultats mathématiques significatifs, et à quelques problèmes ouverts.

Présentation de la chaîne étudiée, sur un exemple simple. Nous considérons des processus markoviens à temps discret, dont les transitions sont sont gouvernées par celles d'une marche aléatoire classique perturbées par une dérive centrifuge. La loi de la marche aléatoire et la perturbation sont soumises à des conditions de symétrie. Ces processus peuvent être à transitions discrètes ou continues, ils peuvent vivre dans un réseau ou dans l'espace euclidien tout entier. Le cadre général est précisément décrit dans la première section de l'article.

Présentons ici l'exemple le plus simple de ces marches aléatoires centrifuges. Nous partons de la marche aléatoire simple sur le réseau $\mathbb{Z}^{d}$, que nous perturbons pour imposer une dérive centrifuge. Pour éviter les formules trop lourdes plaçons nous en dimension $d=2$. Soit $a$ un paramètre réel fixé entre 0 et 1 . La marche centrifuge plane aux plus proches voisins est une chaîne de Markov sur $\mathbb{Z}^{2}$ dont les transitions sont gouvernées par les probabilités suivantes :

$$
P((0,0),(1,0))=P((0,0),(-1,0))=P((0,0),(0,1))=P((0,0),(0,-1))=\frac{1}{4}
$$

et, si $(x, y) \in \mathbb{Z}^{2}$, avec $(x, y) \neq(0,0)$, en notant $\rho:=\sqrt{x^{2}+y^{2}}$,

$$
\begin{aligned}
& P((x, y),(x+1, y))=\frac{1}{4}\left(1+a \frac{x}{\rho}\right), \quad P((x, y),(x-1, y))=\frac{1}{4}\left(1-a \frac{x}{\rho}\right) \\
& P((x, y),(x, y+1))=\frac{1}{4}\left(1+a \frac{y}{\rho}\right), \quad P((x, y),(x, y-1))=\frac{1}{4}\left(1-a \frac{y}{\rho}\right) .
\end{aligned}
$$

L'accroissement moyen d'un pas de cette chaîne à partir du point $(x, y)$ non nul est $\frac{a}{2 \rho}(x, y)$. La dérive est donc centrifuge et d'intensité constante. Pour éviter les problèmes d'arithméticité liés à cet exemple, et pour améliorer l'isotropie de cette marche centrifuge le modèle d'une marche aux huit plus proches voisins a aussi été proposé. En voici une description rapide. L'espace des phases est encore $\mathbb{Z}^{2}$ et on fixe cette fois deux paramètres $a$ et $r$ compris entre 0 et 1 . Les probabilités de transition s'écrivent

$$
\begin{gathered}
P((0,0),(x, y))=\frac{r}{4} \quad \text { si }|x|+|y|=1, \\
P((0,0),(x, y))=\frac{1-r}{4} \quad \text { si }|x|=|y|=1,
\end{gathered}
$$

et, si $(x, y) \in \mathbb{Z}^{2},(x, y) \neq(0,0)$, en notant $\rho:=\sqrt{x^{2}+y^{2}}, \epsilon= \pm 1, \epsilon^{\prime}= \pm 1$,

$$
\begin{gathered}
P((x, y),(x+\epsilon, y))=\frac{r}{4}\left(1+\epsilon a \frac{x}{\rho}\right), \\
P((x, y),(x, y+\epsilon))=\frac{r}{4}\left(1+\epsilon a \frac{y}{\rho}\right), \\
P\left((x, y),\left(x+\epsilon, y+\epsilon^{\prime}\right)\right)=\frac{1-r}{4}\left(1+a \frac{\epsilon x+\epsilon^{\prime} y}{2 \rho}\right) .
\end{gathered}
$$


Méthodes et résultats. Notre objectif est la description du comportement asymptotique de ces chaînes de Markov. Elles sont transientes. Leur vitesse de fuite à l'infini est connue puisque nous démontrons une loi des grands nombres et un théorème limite central pour la norme de la marche. Nous démontrons aussi que, presque sûrement, la marche centrifuge s'échappe à l'infini dans une direction précise, et que norme et direction sont asymptotiquement indépendantes. Les principaux résultats sont rassemblés dans la Section 1.4.

Les théorèmes limites pour martingales constituent notre principal outil. Nous utilisons en particulier un théorème limite central uniforme pour une famille de martingales, que nous présentons en appendice.

Nous laissons ouvertes quelques questions qui nous emblent intéssantes, autour de la loi limite de la direction de la marche et autour du théor 'eme limite local.

Motivation extra-mathématique. Le sujet trouve son origine dans les travaux menés par le premier auteur pour construire dans un espace discret une cinématique à partir de laquelle une machine de Turing pourrait simuler les lois de la physique.

La marche aléatoire centrifuge sur le réseau $\mathbb{Z}^{d}$ a été introduite comme un modéle d'onde circulaire dans un espace physique discret. (Le choix d'un espace discret peut être défendu par plusieurs arguments : remise en cause du dogme de continuité de l'espace ou, plus simplement, nécessité d'utiliser le discret comme approximation du continu pour utiliser l'outil informatique. Les discussions sur ce sujet ne sont pas l'objet de cet article.)

Les résultats décrits dans cette article -vitesse de fuite, isotropie, convergence en loi - confortent le modèle choisi pour représenter l'onde circulaire par un procédé aisément implémentable sur un ordinateur.

\section{LA MARChe CENTRIFUge}

1.1. Présentation du modèle. Soit $d$ un nombre entier $>0$. Nous noterons $<\cdot, \cdot>$ et $\|\cdot\|$ le produit scalaire et la norme usuels de l'espace $\mathbb{R}^{d}$.

Nous nous proposons d'étudier le comportement asymptotique d'une marche aléatoire sur $\mathbb{R}^{d}$ perturbée par une dérive centrifuge. Soit $\mu$ une mesure de probabilité sur $\mathbb{R}^{d}$ qui représentera la loi de la marche non perturbée, et soit $a$ une fonction de $\mathbb{R}^{+}$dans lui-même qui représentera un paramètre de la perturbation. On considère la probabilité de transition définie sur $\mathbb{R}^{d}$ par

$$
P(x, x+\mathrm{d} y):=\left(1+a(\|y\|) \frac{<x, y>}{\|x\|}\right) \mu(\mathrm{d} y) .
$$

(Pour $x=0$ la formule (1) doit se lire simplement $P(0, \mathrm{~d} y)=\mu(\mathrm{d} y)$. Cette convention sera utilisée dans l'ensemble de nos calculs.)

La marche centrifuge est la chaîne de Markov associée à cette probabilité de transition.

Pour que la formule (1) définisse effectivement une probabilité de transition il est nécessaire que pour chaque $x$ dans $\mathbb{R}^{d}$, la fonction $y \mapsto 1+a(\|y\|) \frac{\langle x, y>}{\|x\|}$ soit une densité de probabilité pour la mesure $\mu$. C'est pourquoi nous devons imposer, outre la mesurabilité de la fonction $a$, les deux conditions suivantes

$$
\begin{gathered}
\text { pour } \mu \text {-presque tout } y, \quad\|y\| \cdot a(\|y\|) \leq 1, \\
\int y \cdot a(\|y\|) \mu(\mathrm{d} y)=0 .
\end{gathered}
$$


Nous imposons également à la marche non perturbée d'être non dégénérée, à pas bornés et symétrique par rapport à l'origine. Autrement dit, on suppose que :

(4)

la probabilité $\mu$ est à support borné et est distincte de la masse de Dirac en zéro.

et

$$
\text { pour toute fonction } f \mu \text {-intégrable et impaire, } \quad \int f(y) \mu(\mathrm{d} y)=0 \text {. }
$$

Pour mener à bien notre étude, nous imposons aussi une condition d'isotropie à la marche non perturbée : nous supposons que la variance de la projection de l'accroissement sur une direction quelconque est indépendante de cette direction. Cela se traduit par l'existence d'une constante $m^{\prime}$ telle que, pour tout $\vec{u}$ sur la sphère unité de $\mathbb{R}^{d}$,

$$
m^{\prime}:=\int<y, \vec{u}>^{2} \mu(\mathrm{d} y) .
$$

(Bien sûr, cette condition assure que le support de la marche aléatoire de loi $\mu$ issue de l'origine n'est pas contenu dans un sous-espace vectoriel propre de l'espace $\mathbb{R}^{d}$.)

Enfin, nous imposons les deux conditions suivantes. Si $\vec{u}$ et $\vec{v}$ sont orthogonaux dans $\mathbb{R}^{d}$, alors

$$
\int<y, \vec{u}><y, \vec{v}>a(\|y\|) \mu(\mathrm{d} y)=0
$$

Le nombre

$$
m:=\int<y, \vec{u}>^{2} a(\|y\|) \mu(\mathrm{d} y)
$$

est indépendant du choix de $\vec{u}$ sur la sphère unité de $\mathbb{R}^{d}$.

Compte tenu de l'hypothèse de symétrie (5), la condition (7) indique que l'accroissement moyen de la marche perturbée est centrifuge (ie l'accroissement moyen de la marche issue de $x$ est colinéaire à $x$ ), et la condition (8) indique que l'intensité de cet accroissement est constant (égal à $m$ ).

Nous voulons que la perturbation centrifuge de la marche soit non triviale. Nous excluons donc le cas où, pour $\mu$-presque tout $y \neq 0$, on a $a(\|y\|)=0$. Ainsi on aura toujours $m>0$.

Les conditions (3), (4), (5), (6), (7) et (8) sont évidemment satisfaites par les probabilités invariantes par les isométries vectorielles de $\mathbb{R}^{d}$. Elles le sont aussi, quelque soit le choix de la fonction $a$, par les probabilités discrètes uniformément distribuées sur les vecteurs d'une base orthonormée et leurs opposés. Ces six conditions sont préservées par les similitudes de l'espace vectoriel euclidien $\mathbb{R}^{d}$. Un barycentre (discret ou continu) de probabilités vérifiant ces conditions les vérifie encore.

Exemples. Les exemples présentés dans l'introduction entrent dans ce cadre général. Plaçons nous en dimension $d=2$. Si les transitions de la chaîne se font aux quatre plus proches voisins (on a $\mu=\frac{1}{4}\left(\delta_{(1,0)}+\delta_{(0,1)}+\delta_{(-1,0)}+\delta_{(0,-1)}\right)$ ), alors $m=\frac{1}{2} a(1)$ et $m^{\prime}=\frac{1}{2}$. Si les transitions de la chaîne se font aux huit plus proches voisins (on a

$\mu=\frac{r}{4}\left(\delta_{(1,0)}+\delta_{(0,1)}+\delta_{(-1,0)}+\delta_{(0,-1)}\right)+\frac{1-r}{4}\left(\delta_{(1,1)}+\delta_{(-1,1)}+\delta_{(1,-1)}+\delta_{(-1,-1)}\right)$

où $r$ est un paramètre entre 0 et 1$)$, alors $m=\frac{r}{2} a(1)+(1-r) a(\sqrt{2})$ et $m^{\prime}=1-\frac{r}{2}$. 
1.2. Quelques notations. Notons $\left(X_{n}\right)_{n \geq 0}$ la chaîne de Markov à valeurs dans $\mathbb{R}^{d}$ associée à la probabilité de transition $P$ définie par (1), où la probabilité $\mu$ et la fonction $a$ vérifient l'ensemble des conditions précédentes de (2) à (8). On a, pour toute fonction mesurable bornée $f$ sur $\mathbb{R}^{d}$,

$$
\begin{aligned}
\mathbb{E}\left[f\left(X_{n+1}\right) \mid X_{0}, X_{1}, \ldots, X_{n}\right] & =\mathbb{E}\left[f\left(X_{n+1}\right) \mid X_{n}\right] \\
& =\int f\left(X_{n}+y\right)\left(1+a(\|y\|) \frac{<X_{n}, y>}{\left\|X_{n}\right\|}\right) \mu(\mathrm{d} y) .
\end{aligned}
$$

La loi du processus $\left(X_{n}\right)_{n \geq 0}$ est entièrement déterminée par la loi des transitions et par la distribution $\nu$ de la variable aléatoire $X_{0}$. Nous noterons alors $\mathbb{P}_{\nu}$ la probabilité sur l'espace des réalisations et $\mathbb{E}_{\nu}$ l'espérance associée.

Nous noterons $\rho_{n}:=\left\|X_{n}\right\|$ et $\vec{X}_{n}:=\frac{1}{\rho_{n}} X_{n}$, la norme et la direction du processus.

Si la distribution initiale $\nu$ de la chaîne est une masse de Dirac, c'est-à-dire si la variable aléatoire $X_{0}$ est constante, la probabilité $\mathbb{P}_{\nu}$ sera notée $\mathbb{P}_{\rho_{0}, \vec{X}_{0}}$.

Nous noterons $\mathcal{F}_{n}$ la tribu engendrée par les variables aléatoires ${ }^{\rho_{0}}, X_{1}, \ldots, X_{n}$.

Nous noterons $V:=\int\|y\|^{2} \mu(\mathrm{d} y)$.

Nous noterons enfin $\mathcal{M}^{1}$ l'ensemble des mesures de probabilité sur $\mathbb{R}^{d}$.

1.3. Hypothèses de non-dégénérescence. Avant d'énoncer les principaux résultats décrivant le comportement en temps grand de la marche centrifuge, nous devons distinguer deux situations dégénérées propres à la dimension 1.

- Dans le cas où $d=1$, où la probabilité $\mu$ est portée par deux points $y_{o}>0$ et son opposé $-y_{o}$, et où $a\left(y_{o}\right)=\frac{1}{\left\|y_{o}\right\|}$, la marche centrifuge est déterministe dès qu'elle a quitté l'origine : elle passe de 0 à $\pm y_{o}$ avec probabilité $\frac{1}{2}$, mais elle passe sûrement de $x$ à $x+y_{o}$ pour tout $x>0$, et de $x$ à $x-y_{o}$ pour tout $x<0$. Ce cas particulier sera appelé le premier cas dégénéré.

Les hypothèses (2), (6) et (8), associées à l'inégalité de Cauchy-Schwarz entraînent que, en dehors du premier cas dégénéré, on a toujours $m^{\prime}>m^{2}$. - Dans le cas où $d=1$ et où $a(\|y\|)=\frac{1}{\|y\|}$ pour $\mu$-presque tout $y$ non nul, la marche centrifuge s'éloigne sûrement de l'origine dès qu'elle l'a quittée. Ce cas particulier sera appelé le second cas dégénéré.

\subsection{Principaux résultats.}

Théorème 1. [Loi des grands nombres pour la norme] Quelle que soit la distribution initiale de la chaîne, la suite $\left(\frac{1}{n} \rho_{n}\right)$ tend vers la constante $m$ quand $n$ tend vers l'infini. Plus précisément, pour tout $\epsilon>0$, on a

$$
\lim _{n \rightarrow \infty} n^{1-\epsilon} \mathbb{E}_{\nu}\left[\left(\frac{1}{n}\left(\rho_{n}-\rho_{0}\right)-m\right)^{2}\right]=0 \quad \text { uniformément en } \nu \in \mathcal{M}^{1},
$$

et, $\mathbb{P}_{\nu}$-presque sûrement

$$
\lim _{n \rightarrow \infty} n^{\frac{1}{2}-\epsilon}\left(\frac{1}{n} \rho_{n}-m\right)=0
$$


Théorème 2. [TLC pour la norme] Quelle que soit la distribution initiale de la chaîne, la suite $\left(\frac{1}{\sqrt{n}}\left(\rho_{n}-n m\right)\right)$ converge en loi vers une gaussienne centrée de variance $m^{\prime}-m^{2}$. En dehors du premier cas dégénéré, cette variance est non nulle, et la convergence en loi de $\left(\frac{1}{\sqrt{n}}\left(\rho_{n}-\rho_{0}-n m\right)\right)$ est uniforme en la distribution initiale. Autrement dit, on a

$\lim _{n \rightarrow \infty} \sup _{\nu \in \mathcal{M}^{1}, t \in \mathbb{R}}\left|\mathbb{P}_{\nu}\left[\frac{1}{\sqrt{n\left(m^{\prime}-m^{2}\right)}}\left(\rho_{n}-\rho_{0}-n m\right) \leq t\right]-\frac{1}{\sqrt{2 \pi}} \int_{-\infty}^{t} e^{-s^{2} / 2} \mathrm{~d} s\right|=0$.

Théorème 3. [Convergence de la direction] Quelle que soit la distribution initiale, la suite $\left(\vec{X}_{n}\right)$ converge presque sûrement vers une variable aléatoire $\vec{X}_{\infty}$.

Théorème 4. [Loi limite de la direction] En dehors du second cas dégénéré, et quelle que soit la distribution initiale, le support de la loi de la variable aléatoire $\vec{X}_{\infty}$ est la sphère de $\mathbb{R}^{d}$ toute entière.

Théorème 5. [Indépendance asymptotique de la norme et de la direction] Quelle que soit la distribution initiale, le couple

$$
\left(\frac{\rho_{n}-n m}{\sqrt{n}}, \vec{X}_{n}\right)
$$

converge en loi vers $\left(Z, \vec{X}_{\infty}\right)$, où la variable aléatoire $Z$ est indépendante de $\vec{X}_{\infty}$ et suit la loi gaussienne $\mathcal{N}\left(0, m^{\prime}-m^{2}\right)$.

\section{Comportement en nORMe}

2.1. Estimations de moments. Dans cette section nous donnons des estimations utiles de l'espérance, la variance et la transformée de Laplace du module $\rho_{n}$ de $X_{n}$.

Proposition 1. On a

$$
\mathbb{E}\left[\rho_{n+1}^{2} \mid \mathcal{F}_{n}\right]=\rho_{n}^{2}+2 m \rho_{n}+V
$$

Pour tout $\epsilon>0$, il existe $\alpha>0$ tel que, pour tout $\nu \in \mathcal{M}^{1}$ et pour tout $n \geq 0$,

$$
n m \leq \mathbb{E}_{\nu}\left[\rho_{n}-\rho_{0}\right] \leq n m+\alpha n^{\epsilon} .
$$

On a

$$
\mathbb{E}\left[\rho_{n+1} \mid \mathcal{F}_{n}\right]=\rho_{n}+m+\frac{V-m^{\prime}}{2 \rho_{n}}+O\left(\frac{1}{\rho_{n}^{2}}\right),
$$

où le $O$ est uniforme en l'aléa.

Enfin pour tout $\epsilon>0$, il existe $\beta>0$ tel que, pour tout $\nu \in \mathcal{M}^{1}$ et pour tout $n \geq 0$,

$$
\operatorname{Var}_{\nu}\left[\rho_{n}-\rho_{0}\right] \leq \beta n^{1+\epsilon} .
$$

(On note bien sûr ici $\operatorname{Var}_{\nu}$ la variance sous la probabilité $\mathbb{P}_{\nu}$. Dans les démonstrations, nous utiliserons les notations d'espérance $\mathbb{E}$, de probabilité $\mathbb{P}$ et de variance Var pour des calculs sur la chaîne de Markov avec distribution initiale arbitraire.)

Démonstration. On a

$$
\rho_{n+1}^{2}=\left\|X_{n}+\left(X_{n+1}-X_{n}\right)\right\|^{2}=\rho_{n}^{2}+\left\|X_{n+1}-X_{n}\right\|^{2}+2<X_{n}, X_{n+1}-X_{n}>\text {. }
$$


D'où

$$
\mathbb{E}\left[\rho_{n+1}^{2} \mid \mathcal{F}_{n}\right]=\int\left(\rho_{n}^{2}+\|y\|^{2}+2<X_{n}, y>\right)\left(1+a(\|y\|)<\vec{X}_{n}, y>\right) \mu(\mathrm{d} y) .
$$

En développant cette expression et en utilisant la condition de symétrie (5), on obtient

$$
\mathbb{E}\left[\rho_{n+1}^{2} \mid \mathcal{F}_{n}\right]=\rho_{n}^{2}+\int\|y\|^{2} \mu(\mathrm{d} y)+2 \rho_{n} \int a(\|y\|)<\vec{X}_{n}, y>^{2} \mu(\mathrm{d} y) .
$$

Ce qui est exactement la formule (11).

On a

$$
\rho_{n+1}^{2} \geq \rho_{n}^{2}+\left\|X_{n+1}-X_{n}\right\|^{2}\left(\frac{<X_{n}, X_{n+1}-X_{n}>}{\left\|X_{n}\right\| \cdot\left\|X_{n+1}-X_{n}\right\|}\right)^{2}+2<X_{n}, X_{n+1}-X_{n}>,
$$

c'est-à-dire $\rho_{n+1}^{2} \geq\left(\rho_{n}+<\vec{X}_{n}, X_{n+1}-X_{n}>\right)^{2}$. On en déduit que

$$
\rho_{n+1} \geq \rho_{n}+<\vec{X}_{n}, X_{n+1}-X_{n}>
$$

puis que

$$
\mathbb{E}\left[\rho_{n+1} \mid \mathcal{F}_{n}\right] \geq \int\left(\rho_{n}+<\vec{X}_{n}, y>\right)\left(1+a(\|y\|)<\vec{X}_{n}, y>\right) \mu(\mathrm{d} y) .
$$

En développant cette expression et en utilisant à nouveau la symétrie de la mesure et la formule (8) on obtient

$$
\mathbb{E}\left[\rho_{n+1} \mid \mathcal{F}_{n}\right] \geq \rho_{n}+m
$$

d'où l'on déduit immédiatement la première inégalité de (12).

Démontrons la seconde inégalité de (12). On a

$$
\mathbb{E}\left[\left(\rho_{n+1}-\rho_{0}\right)^{2} \mid \mathcal{F}_{n}\right]=\mathbb{E}\left[\rho_{n+1}^{2} \mid \mathcal{F}_{n}\right]-2 \rho_{0} \mathbb{E}\left[\rho_{n+1} \mid \mathcal{F}_{n}\right]+\rho_{0}^{2} .
$$

En utilisant (11) et (15), on en déduit que

$$
\mathbb{E}\left[\left(\rho_{n+1}-\rho_{0}\right)^{2} \mid \mathcal{F}_{n}\right] \leq \rho_{n}^{2}+2 m \rho_{n}+V-2 \rho_{0}\left(\rho_{n}+m\right)+\rho_{0}^{2} .
$$

ce qui entraîne successivement

$$
\begin{gathered}
\mathbb{E}\left[\left(\rho_{n+1}-\rho_{0}\right)^{2} \mid \mathcal{F}_{n}\right] \leq\left(\rho_{n}-\rho_{0}\right)^{2}+2 m\left(\rho_{n}-\rho_{0}\right)+V, \\
\mathbb{E}\left[\left(\rho_{n+1}-\rho_{0}\right)^{2}\right] \leq \mathbb{E}\left[\left(\rho_{n}-\rho_{0}\right)^{2}\right]+2 m \mathbb{E}\left[\rho_{n}-\rho_{0}\right]+V, \\
\mathbb{E}\left[\left(\rho_{n}-\rho_{0}\right)^{2}\right] \leq 2 m \sum_{k=1}^{n-1} \mathbb{E}\left[\rho_{k}-\rho_{0}\right]+n V,
\end{gathered}
$$

et

$$
\left(\mathbb{E}\left[\rho_{n}-\rho_{0}\right]\right)^{2} \leq 2 m \sum_{k=1}^{n-1} \mathbb{E}\left[\rho_{k}-\rho_{0}\right]+n V .
$$

La seconde inégalité de (12) se déduit alors du lemme suivant.

Lemme 1. Soit $\left(u_{n}\right)_{n \geq 1}$ une suite réelle telle que, pour tout $n$,

$$
u_{n}^{2} \leq 2 m \sum_{k=1}^{n-1} u_{k}+n V .
$$


Alors, pour tout $n$ et tout $\epsilon>0$,

$$
u_{n} \leq n m+\frac{2(1+\epsilon)}{m \epsilon}\left(V-m^{2}\right) n^{\epsilon} .
$$

On aura remarqué, par une simple application de l'inégalité de Cauchy-Schwarz, que $V>m^{2}$. La vérification de ce lemme est laissée au lecteur.

Démontrons à présent l'estimation (13). On a

$$
\mathbb{E}\left[\rho_{n+1} \mid \mathcal{F}_{n}\right]=\int \sqrt{\rho_{n}^{2}+\|y\|^{2}+2<X_{n}, y>}\left(1+a(\|y\|)<\vec{X}_{n}, y>\right) \mu(\mathrm{d} y) .
$$

En utilisant le développement limité

$$
\sqrt{\rho^{2}+\|y\|^{2}+2 \rho<\vec{u}, y>}=\rho+<\vec{u}, y>+\frac{\|y\|^{2}}{2 \rho}-\frac{<\vec{u}, y>^{2}}{2 \rho}+O\left(\frac{1}{\rho^{2}}\right),
$$

(dans lequel le $O$ est uniforme en $\vec{u}$ sur la sphère unité et en $y$ dans une partie bornée de l'espace $\mathbb{R}^{d}$ ), en développant l'intégrale et en utilisant la propriété de symétrie de $\mu$, on obtient bien

$$
\mathbb{E}\left[\rho_{n+1} \mid \mathcal{F}_{n}\right]=\rho_{n}+m+\frac{V}{2 \rho_{n}}-\frac{m^{\prime}}{2 \rho_{n}}+O\left(\frac{1}{\rho_{n}^{2}}\right),
$$

dans lequel le $O$ est uniforme en l'aléa. C'est le résultat annoncé.

Cherchons enfin une estimation de la variance. En utilisant successivement (16) et (12), on peut écrire

$$
\begin{aligned}
\operatorname{Var}\left(\rho_{n}-\rho_{0}\right) & =\mathbb{E}\left[\left(\rho_{n}-\rho_{0}\right)^{2}\right]-\mathbb{E}\left[\rho_{n}-\rho_{0}\right]^{2} \\
& \leq 2 m \sum_{k=1}^{n-1} \mathbb{E}\left[\rho_{k}-\rho_{0}\right]+n V-\mathbb{E}\left[\rho_{n}-\rho_{0}\right]^{2} \\
& \leq 2 m \sum_{k=1}^{n-1}\left(k m+\alpha k^{\epsilon}\right)+n V-(n m)^{2}=\left(V-m^{2}\right) n+2 m \alpha \sum_{k=1}^{n-1} k^{\epsilon} \\
& =O\left(n^{1+\epsilon}\right) .
\end{aligned}
$$

Cela achève la démonstration de la proposition.

Notons sous forme d'un lemme une conséquence utile de la proposition 1.

Lemme 2. Soit $\left(W_{n}\right)$ une suite de variables aléatoires positives définies sur l'espace canonique associé à la chaîne de Markov. S'il existe $c>0$ tel que, pour tout $n$, on ait

$$
W_{n}<c \text { et } W_{n}<c / \rho_{n},
$$

alors, pour tout $\epsilon>0$, il existe $\gamma>0$ tel que, pour tout $\nu \in \mathcal{M}^{1}$ et pour tout $n$,

$$
\mathbb{E}_{\nu}\left[W_{n}\right] \leq \gamma n^{-1+\epsilon} .
$$

Démonstration. On a

$$
\mathbb{P}\left[W_{n}>\frac{2 c}{n m}\right] \leq \mathbb{P}\left[\rho_{n}<\frac{n m}{2}\right] \leq \mathbb{P}\left[\rho_{n}-\rho_{0}<\frac{n m}{2}\right] .
$$


En utilisant l'estimation de l'espérance (12) et l'estimation de variance (14), ainsi que l'inégalité de Bienaymé-Tchebychev, on en déduit que

$$
\begin{aligned}
\mathbb{P}\left[W_{n}>\frac{2 c}{n m}\right] & \leq \mathbb{P}\left[\mathbb{E}\left[\rho_{n}-\rho_{0}\right]-\left(\rho_{n}-\rho_{0}\right)>\frac{n m}{2}\right] \\
& \leq \frac{4}{n^{2} m^{2}} \operatorname{Var}\left[\rho_{n}-\rho_{0}\right] \\
& =O\left(n^{-1+\epsilon}\right),
\end{aligned}
$$

uniformément en la distribution initiale.

Puisque les variables $W_{n}$ sont uniformément bornées par $c$, on a

$$
\mathbb{E}\left[W_{n}\right] \leq c \mathbb{P}\left[W_{n}>\frac{2 c}{n m}\right]+\frac{2 c}{n m} .
$$

Cela permet de conclure que $\mathbb{E}\left[W_{n}\right]=O\left(n^{-1+\epsilon}\right)$, uniformément en la distribution initiale.

Nous noterons $S^{d-1}$ la sphère unité de l'espace euclidien $\mathbb{R}^{d}$.

Proposition 2. Il existe $\lambda>0$ et $\gamma<1$ tels que, pour tout $(\rho, \vec{u}) \in \mathbb{R}^{+} \times S^{d-1}$ et pour tout $n \in \mathbb{N}$,

$$
\mathbb{E}_{\rho, \vec{u}}\left[e^{-\lambda \rho_{n}}\right] \leq \gamma^{n} e^{-\lambda \rho} .
$$

Démonstration. Grâce à la propriété de Markov, il suffit de démontrer qu'il existe $\lambda>0$ et $\gamma<1$ tels que, pour tout $(\rho, \vec{u}) \in \mathbb{R}^{+} \times S^{d-1}$,

$$
\mathbb{E}_{\rho, \vec{u}}\left[e^{-\lambda \rho_{1}}\right] \leq \gamma e^{-\lambda \rho} .
$$

Pour $\vec{u} \in S^{d-1}$, notons $\mu_{\vec{u}}$ la mesure de densité $y \mapsto 1+a(\|y\|)<y, \vec{u}>$ par rapport à la mesure $\mu$. On a

$$
\mathbb{E}_{\rho, \vec{u}}\left[e^{-\lambda\left(\rho_{1}-\rho\right)}\right]=\int e^{-\lambda\left(\sqrt{\rho^{2}+\|y\|^{2}+2 \rho<y, \vec{u}>}-\rho\right)} \mu_{\vec{u}}(\mathrm{~d} y) \leq \int e^{-\lambda<y, \vec{u}>} \mu_{\vec{u}}(\mathrm{~d} y) .
$$

Cette dernière quantité est la transformée de Laplace de $\mu_{\vec{u}}$ évaluée en $\lambda \vec{u}$, que l'on note $L_{\vec{u}}(\lambda)$.

On a $L_{\vec{u}}(0)=1$ et $L_{\vec{u}}^{\prime}(0)=-\int<y, \vec{u}>\mu_{\vec{u}}(\mathrm{~d} y)$. En utilisant (5) et (8), on voit que $L_{\vec{u}}^{\prime}(0)=-m<0$.

On a $L_{\vec{u}}^{\prime \prime}(\lambda)=\int\left\langle y, \vec{u}>^{2} e^{-\lambda<y, \vec{u}>} \mu_{\vec{u}}(\mathrm{~d} y)\right.$, et cette quantité est uniformément bornée par rapport à $\vec{u}$ et $\lambda \geq 0$ puisque le support de $\mu$ est borné. La famille $\left(L_{\vec{u}}^{\prime}\right)$ est donc équicontinue sur $\mathbb{R}^{+}$.

En conséquence, il existe $\lambda>0$ tel que $\gamma:=\sup _{\vec{u} \in S^{d-1}} L_{\vec{u}}(\lambda)<1$. Nous avons établi (17).

\subsection{Loi des grands nombres.}

Démonstration du théorème 1 . Soit $\epsilon>0$. D'après la proposition 1 , on a

$$
\operatorname{Var}\left[\frac{1}{n}\left(\rho_{n}-\rho_{0}\right)\right]=O\left(n^{-1+\epsilon}\right) \quad \text { et } \quad\left|\mathbb{E}\left[\frac{1}{n}\left(\rho_{n}-\rho_{0}\right)\right]-m\right|=O\left(n^{-1+\epsilon}\right) \text {. }
$$


Puisque

$$
\left(\mathbb{E}\left[\left(\frac{1}{n}\left(\rho_{n}-\rho_{0}\right)-m\right)^{2}\right]\right)^{1 / 2} \leq\left(\operatorname{Var}\left[\frac{1}{n}\left(\rho_{n}-\rho_{0}\right)\right]\right)^{1 / 2}+\left|\mathbb{E}\left[\frac{1}{n}\left(\rho_{n}-\rho_{0}\right)\right]-m\right|,
$$

l'affirmation (9) est établie.

Pour établir la convergence ponctuelle avec vitesse, nous allons utiliser un argument de martingale. Posons

$$
Y_{n+1}:=\rho_{n+1}-\mathbb{E}\left[\rho_{n+1} \mid \mathcal{F}_{n}\right] \quad \text { et } \quad Z_{n+1}:=\mathbb{E}\left[\rho_{n+1} \mid \mathcal{F}_{n}\right]-\rho_{n}-m .
$$

La suite $\left(Y_{n}\right)$ est une suite d'accroissements de martingales uniformément bornés,

car $Y_{n+1}=\rho_{n+1}-\rho_{n}-\mathbb{E}\left[\rho_{n+1}-\rho_{n} \mid \mathcal{F}_{n}\right]$. La martingale $\left(\sum_{k=1}^{n} k^{-\frac{1}{2}-\epsilon} Y_{k}\right)$ est bornée en moyenne quadratique, donc presque sûrement convergente. Le lemme de Kronecker permet de conclure que, presque sûrement,

$$
\lim n^{-\frac{1}{2}-\epsilon} \sum_{k=1}^{n} Y_{k}=0
$$

Les variables aléatoires $Z_{n}$ sont uniformément bornées et, d'après (13), il existe une constante $c>0$ telle que, pour tout $n$, on ait $\left|Z_{n}\right|<c / \rho_{n}$. Le lemme 2 permet d'affirmer que $\mathbb{E}\left[\left|Z_{n}\right|\right]=O\left(n^{-1+\epsilon}\right)$. Cela étant vrai pour tout $\epsilon>0$, on en déduit successivement que

$$
\text { la série } \sum_{n \geq 1} \mathbb{E}\left[n^{-\epsilon}\left|Z_{n}\right|\right] \quad \text { est convergente, }
$$

la série $\sum_{n \geq 1} n^{-\epsilon}\left|Z_{n}\right|$ est presque sûrement convergente,

$$
\text { presque sûrement, } \quad \lim n^{-\epsilon} \sum_{k=1}^{n} Z_{k}=0 .
$$

En rassemblant les estimations obtenues sur les séries $\sum Y_{k}$ et $\sum Z_{k}$, on conclut que, presque sûrement,

$$
\rho_{n}-\rho_{0}-n m=\sum_{k=1}^{n}\left(Y_{k}+Z_{k}\right)=o\left(n^{\frac{1}{2}+\epsilon}\right) .
$$

Et on en déduit l'estimation (10).

\subsection{Théorème limite central.}

Démonstration du théorème 2. Cette démonstration est basée sur un théorème limite central uniforme pour martingale dépendant d'un paramètre. Pour une valeur fixée du paramètre, ce TLC est un résultat classique, et nous avons besoin d'une convergence en loi uniforme en le paramètre. Le théorème est précisément décrit en appendice de cet article.

Comme dans la démonstration du théorème 1 , on écrit

$$
\rho_{n}-\rho_{0}-n m=\sum_{k=1}^{n} Y_{k}+\sum_{k=1}^{n} Z_{k}
$$

avec

$$
Y_{n}:=\rho_{n}-\mathbb{E}\left[\rho_{n} \mid \mathcal{F}_{n-1}\right] \quad \text { et } \quad Z_{n}:=\mathbb{E}\left[\rho_{n} \mid \mathcal{F}_{n-1}\right]-\rho_{n-1}-m .
$$


Quelle que soit la distribution initiale $\nu$, la suite $\left(Y_{n}\right)$ est une suite d'accroissements de martingale uniformément bornés par le rayon du support de $\mu$. Notons

$$
V_{n}:=\frac{1}{m^{\prime}-m^{2}} \sum_{k=1}^{n} \mathbb{E}\left[Y_{k}^{2} \mid \mathcal{F}_{k-1}\right]
$$

Nous allons montrer que, pour tout $\delta>0$,

$$
\mathbb{P}_{\nu}\left[\left|1-\frac{V_{n}}{n}\right|>\delta\right] \longrightarrow 0 \text { quand } n \rightarrow \infty \text {, uniformément en } \nu
$$

En utilisant le TLC uniforme (Théorème 6), on en déduit que

$$
\begin{array}{r}
\mathbb{P}_{\nu}\left[\frac{1}{\sqrt{n\left(m^{\prime}-m^{2}\right)}} \sum_{k=1}^{n} Y_{k} \leq t\right]-\frac{1}{\sqrt{2 \pi}} \int_{-\infty}^{t} e^{-s^{2} / 2} \mathrm{~d} s \longrightarrow 0 \\
\text { quand } n \rightarrow \infty, \text { uniformément en }(\nu, t) .
\end{array}
$$

Nous allons montrer également que, pour tout $\delta>0$,

$$
\mathbb{P}_{\nu}\left[\left|\frac{1}{\sqrt{n}} \sum_{k=1}^{n} Z_{k}\right|>\delta\right] \longrightarrow 0 \quad \text { quand } n \rightarrow \infty \text {, uniformément en } \nu \text {. }
$$

En utilisant (19), (20) et l'encadrement

$$
\begin{gathered}
\mathbb{P}_{\nu}\left[\frac{1}{\sqrt{n\left(m^{\prime}-m^{2}\right)}} \sum_{k=1}^{n} Y_{k} \leq t-\delta\right]-\mathbb{P}_{\nu}\left[\frac{1}{\sqrt{n\left(m^{\prime}-m^{2}\right)}} \sum_{k=1}^{n} Z_{k}>\delta\right] \\
\leq \mathbb{P}_{\nu}\left[\frac{1}{\sqrt{n\left(m^{\prime}-m^{2}\right)}}\left(\rho_{n}-\rho_{0}-n m\right) \leq t\right] \leq \\
\mathbb{P}_{\nu}\left[\frac{1}{\sqrt{n\left(m^{\prime}-m^{2}\right)}} \sum_{k=1}^{n} Y_{k} \leq t+\delta\right]+\mathbb{P}_{\nu}\left[\frac{1}{\sqrt{n\left(m^{\prime}-m^{2}\right)}} \sum_{k=1}^{n} Z_{k}<-\delta\right],
\end{gathered}
$$

on obtient sans peine la conclusion du théorème 2 .

Il nous reste à démontrer les affirmations (18) et (20).

De l'identité (13), on déduit que

$$
\mathbb{E}\left[\rho_{n+1} \mid \mathcal{F}_{n}\right]^{2}=\rho_{n}^{2}+2 m \rho_{n}+m^{2}+V-m^{\prime}+O\left(\frac{1}{\rho_{n}}\right),
$$

l'estimation du reste en $O\left(\frac{1}{\rho_{n}}\right)$ étant uniforme en l'aléa. Autrement dit, si l'on pose

$$
R_{n}:=\mathbb{E}\left[\rho_{n+1} \mid \mathcal{F}_{n}\right]^{2}-\left(\rho_{n}^{2}+2 m \rho_{n}+m^{2}+V-m^{\prime}\right),
$$

alors on a $\left|R_{n}\right| \leq \frac{c^{\prime \prime}}{\rho_{n}}$, où $c^{\prime \prime}$ est une constante déterministe. Le lemme 2 s'applique aux variables aléatoires $\left|R_{n}\right|$ : pour tout $\epsilon>0$,

$n^{1-\epsilon} \mathbb{E}\left[\left|R_{n}\right|\right] \longrightarrow 0$ quand $n \rightarrow \infty$, uniformément en la distribution initiale.

La suite $\left(\mathbb{E}\left[R_{n}\right]\right)$ étant uniformément bornée, on en déduit que

$$
n^{-\epsilon} \sum_{k=0}^{n-1} \mathbb{E}\left[\left|R_{k}\right|\right] \longrightarrow 0 \quad \text { quand } n \rightarrow \infty
$$

uniformément en la distribution initiale. 
L'identité (11) s'écrit

$$
\mathbb{E}\left[\rho_{n+1}^{2} \mid \mathcal{F}_{n}\right]=\rho_{n}^{2}+2 m \rho_{n}+V
$$

et nous avons

$$
\mathbb{E}\left[Y_{n+1}^{2} \mid \mathcal{F}_{n}\right]=\mathbb{E}\left[\rho_{n+1}^{2} \mid \mathcal{F}_{n}\right]-\mathbb{E}\left[\rho_{n+1} \mid \mathcal{F}_{n}\right]^{2}
$$

D'où

$$
\mathbb{E}\left[Y_{n+1}^{2} \mid \mathcal{F}_{n}\right]=m^{\prime}-m^{2}-R_{n}
$$

et

$$
1-\frac{V_{n}}{n}=\frac{1}{n\left(m^{\prime}-m^{2}\right)} \sum_{k=0}^{n-1} R_{k}
$$

Ainsi, on a

$$
\mathbb{P}\left[\left|1-\frac{V_{n}}{n}\right|>\delta\right] \leq \frac{1}{n\left(m^{\prime}-m^{2}\right) \delta} \sum_{k=0}^{n-1} \mathbb{E}\left[\left|R_{k}\right|\right],
$$

et de l'estimation (21) découle alors la convergence en probabilité (18).

Pour établir (20), rappelons que la suite $\left(Z_{n} \rho_{n}\right)$ est bornée et que l'on a donc, d'après le lemme 2 ,

$$
\begin{aligned}
\text { pour tout } \epsilon>0, \quad n^{1-\epsilon} \mathbb{E}\left[\left|Z_{n}\right|\right] \longrightarrow 0 \quad \text { quand } n \rightarrow \infty, \\
\text { uniformément en la distribution initiale. }
\end{aligned}
$$

Puisque la suite $\left(\mathbb{E}\left[\left|Z_{n}\right|\right]\right)$ et uniformément bornée, on en déduit que

$$
\frac{1}{\sqrt{n}} \sum_{k=1}^{n} \mathbb{E}\left[\left|Z_{k}\right|\right] \longrightarrow 0 \text { quand } n \rightarrow \infty \text {, uniformément en la distribution initiale. }
$$

Et cela entraîne la propriété de convergence (20). Le théorème 2 est démontré.

\section{Comportement en Direction}

3.1. Convergence. La démonstration de la convergence de la suite $\left(\vec{X}_{n}\right)$ (Théorème 3) est basée sur l'étude de ses accroissements. En norme, ils sont faciles à estimer : puisque le support de la probabilité $\mu$ est borné, il existe une constante réelle $c$ telle que, pour tout $n$,

$$
\left\|\vec{X}_{n+1}-\vec{X}_{n}\right\| \leq \frac{c}{\rho_{n}}
$$

et on sait que, presque sûrement, $\rho_{n}$ est de l'ordre de grandeur de $n$. La moyenne des accroissements est d'un ordre de grandeur inférieur comme nous l'indique le lemme suivant.

Lemme 3. On a $\mathbb{E}\left[\vec{X}_{n+1}-\vec{X}_{n} \mid \mathcal{F}_{n}\right]=O\left(\frac{1}{\rho_{n}^{2}}\right)$, uniformément en l'aléa.

Démonstration. Si $y=X_{n+1}-X_{n}$, alors $\rho_{n+1}=\sqrt{\rho_{n}^{2}+2 \rho_{n}<y, \vec{X}_{n}>+\|y\|^{2}}$. D'où 


$$
\begin{aligned}
& \mathbb{E}\left[\vec{X}_{n+1}-\vec{X}_{n} \mid \mathcal{F}_{n}\right]= \\
& \int\left(\frac{\rho_{n} \vec{X}_{n}+y}{\sqrt{\rho_{n}^{2}+2 \rho_{n}<y, \vec{X}_{n}>+\|y\|^{2}}}-\vec{X}_{n}\right)\left(1+a(\|y\|)<y, \vec{X}_{n}>\right) \mu(\mathrm{d} y)= \\
& \int\left(\frac{\rho_{n} \vec{X}_{n}+y}{\rho_{n}\left(1+<y, \vec{X}_{n}>\rho_{n}^{-1}+O\left(\rho_{n}^{-2}\right)\right)}-\vec{X}_{n}\right)\left(1+a(\|y\|)<y, \vec{X}_{n}>\right) \mu(\mathrm{d} y)= \\
& \frac{1}{\rho_{n}} \int\left(y-<y, \vec{X}_{n}>\vec{X}_{n}+O\left(\rho_{n}^{-1}\right)\right)\left(1+a(\|y\|)<y, \vec{X}_{n}>\right) \mu(\mathrm{d} y) .
\end{aligned}
$$

Nous savons que la densité $y \mapsto 1+a(\|y\|)<y, \vec{X}_{n}>$ est bornée. On a donc

$$
\begin{aligned}
& \mathbb{E}\left[\vec{X}_{n+1}-\vec{X}_{n} \mid \mathcal{F}_{n}\right]= \\
& \quad \frac{1}{\rho_{n}} \int\left(y-<y, \vec{X}_{n}>\vec{X}_{n}\right)\left(1+a(\|y\|)<y, \vec{X}_{n}>\right) \mu(\mathrm{d} y)+O\left(\rho_{n}^{-2}\right) .
\end{aligned}
$$

Montrons que l'intégrale apparaissant dans cette dernière expression est nulle. Cela concluera la démonstration du lemme.

D'une part, puisque la mesure $\mu$ est symétrique, on a

$$
\int\left(y-<y, \vec{X}_{n}>\vec{X}_{n}\right) \mu(\mathrm{d} y)=0
$$

D'autre part, la condition (7) nous dit que, pour tout $\vec{u}$, le vecteur $\int<y, \vec{u}>a(\|y\|) \cdot y \mu(\mathrm{d} y)$ est colinéaire à $\vec{u}$. Ainsi, pour tout $\vec{u} \in S^{d-1}$, on a

$$
\int<y, \vec{u}>a(\|y\|) \cdot y \mu(\mathrm{d} y)=\int<y, \vec{u}>^{2} a(\|y\|) \cdot \vec{u} \mu(\mathrm{d} y),
$$

ce qui nous donne bien

$$
\int\left(y-<y, \vec{X}_{n}>\vec{X}_{n}\right) a(\|y\|)<y, \vec{X}_{n}>\mu(\mathrm{d} y)=0 .
$$

Démonstration du théorème 3. Le lemme 3, associé à la proposition 1, nous donne

$$
\text { presque sûrement, } \quad \mathbb{E}\left[\vec{X}_{n+1}-\vec{X}_{n} \mid \mathcal{F}_{n}\right]=O\left(n^{-2}\right) \text {. }
$$

Posons

$$
\Delta_{n+1}:=\vec{X}_{n+1}-\mathbb{E}\left[\vec{X}_{n+1} \mid \mathcal{F}_{n}\right]=\vec{X}_{n+1}-\vec{X}_{n}-\mathbb{E}\left[\vec{X}_{n+1}-\vec{X}_{n} \mid \mathcal{F}_{n}\right] .
$$


La suite $\left(\Delta_{n}\right)$ est une suite (vectorielle) d'accroissements de martingale et, grâce à (22), on a

$$
\begin{aligned}
\mathbb{E}\left[\left\|\Delta_{n+1}\right\|^{2} \mid \mathcal{F}_{n}\right] & =\mathbb{E}\left[\left\|\vec{X}_{n+1}-\vec{X}_{n}\right\|^{2} \mid \mathcal{F}_{n}\right]-\left\|\mathbb{E}\left[\vec{X}_{n+1}-\vec{X}_{n} \mid \mathcal{F}_{n}\right]\right\|^{2} \\
& \leq \mathbb{E}\left[\left\|\vec{X}_{n+1}-\vec{X}_{n}\right\|^{2} \mid \mathcal{F}_{n}\right] \\
& \leq \frac{c^{2}}{\rho_{n}^{2}} .
\end{aligned}
$$

On déduit du théorème 1 que, presque sûrement,

$$
\sum_{n=0}^{+\infty} \mathbb{E}\left[\left\|\Delta_{n+1}\right\|^{2} \mid \mathcal{F}_{n}\right]<+\infty .
$$

Un théorème classique de convergence de martingales (cf par exemple [6], Proposition IV-6-2), nous permet alors d'affirmer que la série $\sum_{n=1}^{+\infty} \Delta_{n}$ converge presque sûrement. Or l'estimation (23) assure la convergence presque sûre de la série $\sum_{n=0}^{+\infty} \mathbb{E}\left[\vec{X}_{n+1}-\vec{X}_{n} \mid \mathcal{F}_{n}\right]$. On conclut que la série de terme général $\vec{X}_{n+1}-\vec{X}_{n}$ converge presque sûrement. C'est ce qu'il fallait démontrer.

3.2. Piégeage dans les cônes et support de la loi limite. La proposition suivante nous dit que si la marche a atteint un point éloigné de l'origine dans un cône ouvert, alors elle restera définitivement dans ce cône avec une forte probabilité.

Proposition 3. Pour tout $\epsilon>0$, on a

$$
\lim _{\rho \rightarrow \infty} \sup _{\vec{u} \in S^{d-1}}\left(1-\mathbb{P}_{\rho, \vec{u}}\left[\forall n \geq 1,\left\|\vec{X}_{n}-\vec{u}\right\|<\epsilon\right]\right)=0 .
$$

Pour démontrer cette proposition, nous utiliserons le lemme suivant, conséquence de la proposition 2 .

Lemme 4. Il existe $\alpha>0$ tel que, pour tout $\beta>0$, il existe $C_{\beta}>0$ tel que, pour tout $(\rho, \vec{u}) \in \mathbb{R}^{+} \times S^{d-1}$ et tout $n \geq 0$,

$$
\mathbb{E}_{\rho, \vec{u}}\left[\frac{1}{\left(1+\rho_{n}\right)^{\beta}}\right] \leq \frac{C_{\beta}}{(1+n \alpha+\sqrt{\rho})^{\beta}} .
$$

Démonstration. Nous utilisons les notations introduites dans la proposition 2, et fixons $\alpha>0$ assez petit pour que $r_{\alpha}:=\gamma e^{\alpha \lambda}<1$. On a $\mathbb{E}_{\rho, \vec{u}}\left[e^{-\lambda\left(\rho_{n}-n \alpha\right)}\right] \leq r_{\alpha}^{n} e^{-\lambda \rho}$, et

$$
\begin{aligned}
\mathbb{E}_{\rho, \vec{u}}\left[\frac{1}{\left(1+\rho_{n}\right)^{\beta}}\right] & \leq \mathbb{P}_{\rho, \vec{u}}\left[\rho_{n}<n \alpha+\sqrt{\rho}\right]+\mathbb{E}_{\rho, \vec{u}}\left[\frac{1}{\left(1+\rho_{n}\right)^{\beta}}, \rho_{n} \geq n \alpha+\sqrt{\rho}\right] \\
& \leq \mathbb{E}_{\rho, \vec{u}}\left[e^{-\lambda\left(\rho_{n}-n \alpha-\sqrt{\rho}\right)}\right]+\frac{1}{(1+n \alpha+\sqrt{\rho})^{\beta}} \\
& \leq r_{\alpha}^{n} e^{-\lambda(\rho-\sqrt{\rho})}+\frac{1}{(1+n \alpha+\sqrt{\rho})^{\beta}} .
\end{aligned}
$$


Pour conclure la démonstration du lemme, il suffit de remarquer qu'il existe un nombre $C>0$, indépendant de $n \in \mathbb{N}$ et de $\rho \in \mathbb{R}^{+}$tel que

$$
r_{\alpha}^{n} e^{-\lambda(\rho-\sqrt{\rho})} \leq \frac{C}{(1+n \alpha+\sqrt{\rho})^{\beta}} .
$$

Démonstration de la proposition 3. Comme dans la démonstration du théorème 3 nous posons

et nous avons

$$
\Delta_{k}:=\vec{X}_{k}-\mathbb{E}\left[\vec{X}_{k} \mid \mathcal{F}_{k-1}\right],
$$

$$
\vec{X}_{n}-\vec{X}_{0}=\sum_{k=1}^{n} \Delta_{k}+\sum_{k=1}^{n} \mathbb{E}\left[\vec{X}_{k}-\vec{X}_{k-1} \mid \mathcal{F}_{k-1}\right] .
$$

Nous utiliserons l'inégalité

$$
\sup _{n \geq 1}\left\|\vec{X}_{n}-\vec{X}_{0}\right\| \leq \sup _{n \geq 1}\left\|\sum_{k=1}^{n} \Delta_{k}\right\|+\sum_{k=1}^{+\infty}\left|\mathbb{E}\left[\vec{X}_{k}-\vec{X}_{k-1} \mid \mathcal{F}_{k-1}\right]\right| .
$$

L'inégalité maximale de Kolmogorov appliquée à la martingale $\left(\sum_{k=1}^{n} \Delta_{k}\right)_{n \geq 1}$ nous donne (quelle que soit la distribution initiale) :

$$
\mathbb{P}\left[\max _{1 \leq m \leq n}\left\|\sum_{k=1}^{m} \Delta_{k}\right\| \geq \frac{\epsilon}{2}\right] \leq \frac{4}{\epsilon^{2}} \mathbb{E}\left[\left\|\sum_{k=1}^{n} \Delta_{k}\right\|^{2}\right]=\frac{4}{\epsilon^{2}} \sum_{k=1}^{n} \mathbb{E}\left[\left\|\Delta_{k}\right\|^{2}\right] .
$$

La majoration (22) garantit l'existence d'une constante $c^{\prime}$ telle que,

$$
\mathbb{E}\left[\left\|\Delta_{k}\right\|^{2}\right] \leq \mathbb{E}\left[\left\|\vec{X}_{k}-\vec{X}_{k-1}\right\|^{2}\right] \leq \mathbb{E}\left[\frac{c^{\prime}}{\left(1+\rho_{k-1}\right)^{2}}\right]
$$

D'où

$$
\mathbb{P}\left[\max _{1 \leq m \leq n}\left\|\sum_{k=1}^{m} \Delta_{k}\right\| \geq \frac{\epsilon}{2}\right] \leq \frac{4 c^{\prime}}{\epsilon^{2}} \sum_{k=0}^{n-1} \mathbb{E}\left[\frac{1}{\left(1+\rho_{k}\right)^{2}}\right] .
$$

En faisant tendre $n$ vers l'infini, on arrive à

$$
\mathbb{P}\left[\sup _{m \geq 1}\left\|\sum_{k=1}^{m} \Delta_{k}\right\| \geq \frac{\epsilon}{2}\right] \leq \frac{4 c^{\prime}}{\epsilon^{2}} \sum_{k=0}^{+\infty} \mathbb{E}\left[\frac{1}{\left(1+\rho_{k}\right)^{2}}\right] .
$$

Par ailleurs, grâce au lemme 3 , on sait qu'il existe une constante $c^{\prime \prime}$, indépendante de la distribution initiale telle que

Ainsi,

$$
\mathbb{E}\left[\left\|\mathbb{E}\left[\vec{X}_{k}-\vec{X}_{k-1} \mid \mathcal{F}_{k-1}\right]\right\|\right] \leq c^{\prime \prime} \mathbb{E}\left[\frac{1}{\left(1+\rho_{k-1}\right)^{2}}\right]
$$

$$
\sum_{k=1}^{+\infty} \mathbb{E}\left[\left\|\mathbb{E}\left[\vec{X}_{k}-\vec{X}_{k-1} \mid \mathcal{F}_{k-1}\right]\right\|\right] \leq c^{\prime \prime} \sum_{k=0}^{+\infty} \mathbb{E}\left[\frac{1}{\left(1+\rho_{k}\right)^{2}}\right]
$$

d'où

$$
\mathbb{P}\left[\sum_{k=1}^{+\infty}\left|\mathbb{E}\left[\vec{X}_{k}-\vec{X}_{k-1} \mid \mathcal{F}_{k-1}\right]\right| \geq \frac{\epsilon}{2}\right] \leq \frac{2 c^{\prime \prime}}{\epsilon} \sum_{k=0}^{+\infty} \mathbb{E}\left[\frac{1}{\left(1+\rho_{k}\right)^{2}}\right] .
$$


Cette inégalité, associée à (24) et (25), nous donne l'existence d'une constante $c_{\epsilon}$, indépendante de la distribution initiale telle que

$$
\mathbb{P}\left[\sup _{n \geq 1}\left\|\vec{X}_{n}-\vec{X}_{0}\right\| \geq \epsilon\right] \leq c_{\epsilon} \sum_{k=0}^{+\infty} \mathbb{E}\left[\frac{1}{\left(1+\rho_{k}\right)^{2}}\right] .
$$

En utilisant le lemme 4, on en déduit l'existence d'une constante $c_{\epsilon}^{\prime}$ telle que, pour tout $(\rho, \vec{u})$,

$$
\mathbb{P}_{\rho, \vec{u}}\left[\sup _{n \geq 1}\left\|\vec{X}_{n}-\vec{X}_{0}\right\| \geq \epsilon\right] \leq c_{\epsilon}^{\prime} \sum_{k=0}^{n} \frac{1}{(1+k \alpha+\sqrt{\rho})^{2}}
$$

et cette dernière quantité tend vers zéro quand $\rho$ tend vers l'infini.

Démonstration du théorème 4. Nous devons démontrer que, pour tout ouvert non vide $O$ de la sphère $S^{d-1}$ (et quelle que soit la distribution initiale) on a

$$
\mathbb{P}\left[\vec{X}_{\infty} \in O\right]>0
$$

Il suffit de démontrer que, pour tout ouvert non vide $O$ de la sphère $S^{d-1}$, il existe $n_{0} \geq 0$ tel que, $\mathbb{P}\left[\forall n \geq n_{0}, \vec{X}_{n} \in O\right]>0$.

Fixons un tel ouvert $O$ et considérons $\vec{u} \in O$ et $\epsilon>0$ tels que

$$
\vec{v} \in S^{d-1} \text { et }\|\vec{v}-\vec{u}\| \leq 2 \epsilon \Rightarrow \vec{v} \in O
$$

La proposition 3 nous dit que si la marche passe par un point assez éloigné de l'origine, elle restera ensuite définitivement dans un petit cône centré en ce point avec une probabilité non nulle : il existe $r>0$ tel que si $\rho>r$ et si $\vec{v} \in S^{d-1}$, alors $\mathbb{P}_{\rho, \vec{v}}\left[\forall n \geq 1,\left\|\vec{X}_{n}-\vec{v}\right\| \leq \epsilon\right]>0$.

Si pour un entier $n_{0}$ on a $\mathbb{P}\left[\left\|\vec{X}_{n_{0}}-\vec{u}\right\|<\epsilon\right.$ et $\left.\rho_{n_{0}} \geq r\right]>0$, alors, grâce à la propriété de Markov on obtient $\mathbb{P}\left[\forall n \geq n_{0},\left\|\vec{X}_{n}-\vec{u}\right\| \leq 2 \epsilon\right]>0$.

Il nous suffit donc de démontrer que, quelle que soit la distribution initiale, la marche visite avec une probabilité non nulle le domaine

$$
\left\{x \in \mathbb{R}^{d} \mid\|x\| \geq r \text { et }\left\|\frac{x}{\|x\|}-\vec{u}\right\| \leq \epsilon\right\} .
$$

C'est une conséquence du lemme suivant, qui concluera la démonstration du théorème 4 .

Lemme 5. Il existe $R>0$ tel que, quelle que soit la distribution initiale, toute boule de rayon $R$ est visitée avec probabilité non nulle par la marche aléatoire centrifuge $\left(X_{n}\right)$.

Démonstration. Commençons par remarquer que tout sous-groupe additif de $\mathbb{R}^{d}$ qui n'est pas contenu dans un sous-espace vectoriel propre rencontre toute boule de rayon suffisamment grand.

Le support de la mesure $\mu$ est symétrique par rapport à zéro et n'est pas contenu dans un sous-espace vectoriel propre de $\mathbb{R}^{d}$. Le support de la marche aléatoire de loi $\mu$ issue de l'origine 0 est donc un sous-groupe $G$ de $\mathbb{R}^{d}$, et il n'est pas contenu dans un sous-espace vectoriel propre de cet espace. Le support de la marche aléatoire de loi $\mu$ et de distribution initiale arbitraire fixée est une réunion de translatés de $G$. 
Toute boule de rayon suffisamment grand est donc visitée par la marche aléatoire de loi $\mu$ avec une probabilité non nulle.

Étudions à présent la marche centrifuge. Pour chaque $x$ dans $\mathbb{R}^{d}$, le support de la probabilité $\mathbb{P}(x, \cdot)$ est contenu dans celui de la probabilité $\mu$ translatée par $x$. Si, pour chaque $x$ dans $\mathbb{R}^{d}$, le support de la probabilité $\mathbb{P}(x, \mathrm{~d} y)$ est le même que celui de la probabilité $x+\mu(\mathrm{d} y)$, alors le support de la marche centrifuge est le même que celui de la marche de loi $\mu$ et le lemme découle de l'affirmation de l'alinéa précédent.

Mais il peut arriver que le support de la probabilité $\mathbb{P}(x, \cdot)$ ne soit pas le même que celui de la probabilité $\delta_{x} \star \mu$. On remarque tout de même que le support de la probabilité $\mathbb{P}(x, \cdot)$ contient le support de $\delta_{x} \star \mu$ privé des points $z$ placés sur la demi-droite issue de $x$ et passant par l'origine. En effet, c'est seulement en ces points $z$ que la densité de $\mathbb{P}(x, \cdot)$ par rapport à $\delta_{x} \star \mu$ peut s'annuler.

Dans le cas $d=1$ et en dehors du premier cas dégénéré, il est facile de voir que le support de la marche centrifuge rencontre tous les intervalles suffisamment longs.

En dimension $d \geq 2$, en utilisant le fait que le support de la mesure $\mu$ est symétrique par rapport à l'origine et non contenu dans une droite, on peut montrer que

$$
\begin{aligned}
& \text { le support de la marche centrifuge est le même que celui de } \\
& \qquad \text { la marche aléatoire de loi } \mu \text {, privé éventuellement de l'origine. }
\end{aligned}
$$

Cela suffit pour conclure comme précédemment et achever la démonstration.

Il nous reste donc à justifier l'affirmation (26). Pour des raisons de facilité de rédaction, nous nous limiterons à l'exposé du cas où la mesure $\mu$ est discrète.

Pour tout nombre entier $n \geq 1$, notons $\mathbb{P}^{(n)}(x, y)$ la probabilité de transition en $n$ pas de la marche centrifuge. Deux points $x$ et $y$ de l'espace sont connectables par la marche centrifuge si et seulement si il existe $n$ tel que $\mathbb{P}^{(n)}(x, y)>0$. Ces deux points sont connectables par la marche aléatoire de loi $\mu$ si et seulement si il existe $n$ tel que $\mu^{* n}(y-x)>0$. Nous voulons démontrer que si les points $x$ et $y$ sont connectables par la marche aléatoire de loi $\mu$ et si $y$ n'est pas l'origine, alors ces points sont connectables par la marche centrifuge. Examinons plusieurs situations.

- Si $x, y$ et l'origine ne sont pas alignés et si $\mu(y-x)>0$, alors $\mathbb{P}(x, y)>0$. Cela a déjà été vu.

- Si $x, y$ et l'origine sont alignés et deux à deux distincts et si $\mu(y-x)>0$, alors $\mathbb{P}^{(3)}(x, y)>0$. En effet, le support de $\mu$ n'étant pas contenu dans une droite, on peut considérer un point $z$ non aligné avec $x, y$ et 0 tel que $\mu(z-x)>0$. On a $\mu((z+y-x)-z)>0$ et $\mu(y-(z+y-x))>0$. Comme de plus aucun des trois triplets de points $(x, z, 0),(z, z+y-x, 0),(z+y-x, y, 0)$ n'est un triplet de points alignés, on a $P(x, z)>0, P(z, z+y-x)>0$ et $P(z+y-x, y)>0$. D'où $P^{(3)}(x, y)>0$.

- Pour chaque $y$, on a $P(0, y)=\mu(y)$. Ainsi, si $x=0$ et $\mu(y-x)>0$, alors $P(x, y)>0$.

(bilan d'étape : nous savons que si la marche aléatoire de loi $\mu$ permet de passer de $x$ à $y$ en un pas et si $y \neq 0$, alors la marche centrifuge permet de passer de $x$ à $y$, en un ou trois pas ; il nous reste à examiner ce qui se passe pour deux points que la marche aléatoire de loi $\mu$ connecte en passant par l'origine.) 
- Soient deux points $x$ et $y$ non alignés avec l'origine et tels que $\mu(-x)>0$ et $\mu(y)>0$. On a $\mu((x+y)-x)>0$ et $\mu(y-(x+y))>0$. Comme aucun des deux triplets de points $(x, x+y, 0)$ et $(x+y, y, 0)$ n'est un triplet de points alignés, on a $P(x, x+y)>0$ et $P(x+y, y)>0$. D'où $P^{(2)}(x, y)>0$.

- Soient enfin deux points $x$ et $y$ alignés avec l'origine et distincts de celle-ci, tels que $\mu(-x)>0$ et $\mu(y)>0$. Il existe un point $z$, hors de la droite portant $x, y$ et 0 , tel que $\mu(z-x)>0$. On a $\mu((z-x)-z)>0, \mu((z-x+y)-(z-x))>$ 0 et $\mu(y-(z-x+y))>0$. Comme aucun des quatre triplets de points $(x, z, 0),(z, z-x, 0),(z-x, z-x+y, 0)$ et $(z-x+y, y, 0)$ n'est un triplet de points alignés, on a $P(x, z)>0, P(z, z-x)>0, P(z-x, z-x+y)>0$ et $P(z-x+y, y)>0$. D'où $P^{(4)}(x, y)>0$.

L'examen de l'ensemble de ces cas nous montre que :

- À chaque pas de la marche aléatoire de loi $\mu$ qui relie un point quelconque à un point distinct de l'origine on peut associer une trajectoire de la marche centrifuge qui possède les mêmes extrémités.

- À chaque paire de pas de la marche aléatoire de loi $\mu$ qui relie deux points distincts de l'origine en passant par l'origine, on peut associer une trajectoire de la marche centrifuge qui possède les mêmes extrémités.

Ainsi, pour toute trajectoire de la marche aléatoire de loi $\mu$ relie une origine $x$ à une extrémité $y$ distincte de l'origine, il existe une trajectoire de la marche centrifuge qui possède les mêmes extrémités. Cela justifie l'affirmation (26).

\section{IndÉPENDANCE ASYMPTOTIQUE DE LA NORME ET DE LA DIRECTION}

L'objet de cette section est la démonstration de l'indépendance asymptotique des variables aléatoires $\rho_{n}$ (convenablement normalisée) et $\vec{X}_{n}$.

Démonstration du théorème 5 . On considère la marche centrifuge de distribution initiale arbitraire et fixée $\nu$, et on note $\mathbb{P}=\mathbb{P}_{\nu}$. Pour démontrer le théorème, il suffit de démontrer que, si $t \in \mathbb{R}$ et si $A$ est un borélien de la sphère $S^{d-1}$ de frontière négligeable pour la loi de $\vec{X}_{\infty}$ (ie $\mathbb{P}\left[\vec{X}_{\infty} \in \partial A\right]=0$ ), alors

$$
\begin{aligned}
\lim _{n \rightarrow \infty} \mathbb{P}\left[\rho_{n} \leq n m+t \sqrt{n\left(m^{\prime}-m^{2}\right)} \text { et } \vec{X}_{n} \in A\right]= & \\
& \left(\frac{1}{\sqrt{2 \pi}} \int_{-\infty}^{t} e^{-s^{2} / 2} \mathrm{~d} s\right) \cdot \mathbb{P}\left[\vec{X}_{\infty} \in A\right] .
\end{aligned}
$$

(Les caractérisations classiques de la convergence en loi sont exposées par exemple dans [1].)

Fixons $t$ et $A$. Posons $\left.I_{n}:=\right]-\infty, n m+t \sqrt{n\left(m^{\prime}-m^{2}\right)}$ ]. Si $B$ est un sousensemble de $S^{d-1}$, nous noterons $B^{c}$ son complémentaire et, pour $\epsilon>0$, nous noterons $B_{\epsilon}$ le $\epsilon$-voisinage de $B$ dans $S^{d-1}$, défini par

$$
B_{\epsilon}:=\left\{\vec{u} \in S^{d-1}: \exists \vec{v} \in B,\|\vec{u}-\vec{v}\|<\epsilon\right\} .
$$

Fixons deux suites de nombres entiers positifs $\left(a_{n}\right)$ et $\left(b_{n}\right)$ telles que, quand $n \rightarrow \infty$,

$$
b_{n} \rightarrow \infty, \quad \frac{a_{n}}{b_{n}} \rightarrow \infty, \quad a_{n}<n \quad \text { et } \quad \frac{\sqrt{n}}{a_{n}} \rightarrow \infty .
$$

Enfin, nous noterons $\mathcal{L}_{\rho_{n}, \vec{X}_{n}}$ la loi du couple de variables aléatoires $\left(\rho_{n}, \vec{X}_{n}\right)$. 
La formule de Chapman-Kolmogorov permet d'écrire

$$
\begin{aligned}
& \mathbb{P}\left[\rho_{n} \in I_{n} \text { et } \vec{X}_{n} \in A\right]= \\
& \qquad \int_{\mathbb{R}^{+} \times S^{d-1}} \mathbb{P}_{\rho, \vec{u}}\left[\rho_{n-a_{n}} \in I_{n} \text { et } \vec{X}_{n-a_{n}} \in A\right] \mathrm{d} \mathcal{L}_{\rho_{a_{n}}, \vec{X}_{a_{n}}}(\rho, \vec{u}) .
\end{aligned}
$$

On décompose cette expression en trois parties

$$
\mathbb{P}\left[\rho_{n} \in I_{n} \text { et } \vec{X}_{n} \in A\right]=\alpha_{n}-\beta_{n}+\gamma_{n},
$$

où

$$
\begin{aligned}
\alpha_{n} & :=\int_{\mathbb{R}^{+} \times A^{c}} \mathbb{P}_{\rho, \vec{u}}\left[\rho_{n-a_{n}} \in I_{n} \text { et } \vec{X}_{n-a_{n}} \in A\right] \mathrm{d} \mathcal{L}_{\rho_{a_{n}}, \vec{X}_{a_{n}}}(\rho, \vec{u}), \\
\beta_{n} & :=\int_{\mathbb{R}^{+} \times A} \mathbb{P}_{\rho, \vec{u}}\left[\rho_{n-a_{n}} \in I_{n} \text { et } \vec{X}_{n-a_{n}} \notin A\right] \mathrm{d} \mathcal{L}_{\rho_{a_{n}}, \vec{X}_{a_{n}}}(\rho, \vec{u}),
\end{aligned}
$$

et

$$
\gamma_{n}:=\int_{\mathbb{R}^{+} \times A} \mathbb{P}_{\rho, \vec{u}}\left[\rho_{n-a_{n}} \in I_{n}\right] \mathrm{d} \mathcal{L}_{\rho_{a_{n}}, \vec{X}_{a_{n}}}(\rho, \vec{u}) .
$$

Pour étudier la quantité $\alpha_{n}$, on introduit un paramètre $\epsilon>0$ et on découpe le domaine d'intégration en excluant les événements $\left[\rho_{a_{n}} \leq b_{n}\right]$ et $\left[\vec{X}_{a_{n}} \in A_{\epsilon}\right]$. On obtient la majoration suivante :

$$
\begin{aligned}
\alpha_{n} \leq \mathbb{P}\left[\rho_{a_{n}} \leq b_{n}\right]+\mathbb{P}\left[\vec{X}_{a_{n}} \in A_{\epsilon} \backslash A\right]+ & \\
& \int_{] b_{n},+\infty\left[\times\left(A_{\epsilon}\right)^{c}\right.} \mathbb{P}_{\rho, \vec{u}}\left[\vec{X}_{n-a_{n}} \in A\right] \mathrm{d} \mathcal{L}_{\rho_{a_{n}}, \vec{X}_{a_{n}}}(\rho, \vec{u}) .
\end{aligned}
$$

D'après le théorème 1 , on a $\lim \mathbb{P}\left[\rho_{a_{n}} \leq b_{n}\right]=0$.

Le théorème 3 assure en particulier la convergence en loi de la suite $\left(\vec{X}_{a_{n}}\right)$ vers $\vec{X}_{\infty}$, et on en déduit que

$$
\limsup \mathbb{P}\left[\vec{X}_{a_{n}} \in A_{\epsilon} \backslash A\right] \leq \limsup \mathbb{P}\left[\vec{X}_{a_{n}} \in \overline{A_{\epsilon} \backslash A}\right] \leq \mathbb{P}\left[\vec{X}_{\infty} \in \overline{A_{\epsilon} \backslash A}\right] .
$$

Enfin, puisque $\bigcap_{\epsilon>0} \overline{A_{\epsilon} \backslash A}=\partial A$ on a $\lim _{\epsilon \rightarrow 0} \mathbb{P}\left[\vec{X}_{\infty} \in \overline{A_{\epsilon} \backslash A}\right]=\mathbb{P}\left[\vec{X}_{\infty} \in \partial A\right]=0$.

Le troisième terme de la majoration de $\alpha_{n}$ est une intégrale sur un domaine où la variable $\vec{u}$ reste à distance $\geq \epsilon$ de l'ensemble $A$. Forts de cette observation, nous écrivons

$$
\begin{aligned}
\int_{] b_{n},+\infty\left[\times\left(A_{\epsilon}\right)^{c}\right.} \mathbb{P}_{\rho, \vec{u}}\left[\vec{X}_{n-a_{n}} \in A\right] \mathrm{d} \mathcal{L}_{\rho_{a_{n}}, \vec{X}_{a_{n}}}(\rho, \vec{u}) \leq \\
\int_{] b_{n},+\infty\left[\times\left(A_{\epsilon}\right)^{c}\right.} \mathbb{P}_{\rho, \vec{u}}\left[\left\|\vec{X}_{n-a_{n}}-\vec{u}\right\| \geq \epsilon\right] \mathrm{d} \mathcal{L}_{\rho_{a_{n}}, \vec{X}_{a_{n}}}(\rho, \vec{u}),
\end{aligned}
$$

puis

$$
\begin{aligned}
& \int_{] b_{n},+\infty\left[\times\left(A_{\epsilon}\right)^{c}\right.} \mathbb{P}_{\rho, \vec{u}}\left[\vec{X}_{n-a_{n}} \in A\right] \mathrm{d} \mathcal{L}_{\rho_{a_{n}}, \vec{X}_{a_{n}}}(\rho, \vec{u}) \leq \\
& \sup _{\rho>b_{n}, \vec{u} \in S^{d-1}} \mathbb{P}_{\rho, \vec{u}}\left[\left\|\vec{X}_{n-a_{n}}-\vec{u}\right\| \geq \epsilon\right] .
\end{aligned}
$$


Enfin

$$
\begin{aligned}
& \int_{] b_{n},+\infty\left[\times\left(A_{\epsilon}\right)^{c}\right.} \mathbb{P}_{\rho, \vec{u}}\left[\vec{X}_{n-a_{n}} \in A\right] \mathrm{d} \mathcal{L}_{\rho_{a_{n}}, \vec{X}_{a_{n}}}(\rho, \vec{u}) \leq \\
& \sup _{\rho>b_{n}, \vec{u} \in S^{d-1}} \mathbb{P}_{\rho, \vec{u}}\left[\exists k>0,\left\|\vec{X}_{k}-\vec{u}\right\| \geq \epsilon\right] .
\end{aligned}
$$

D'après la proposition 3 , cette dernière quantité tend vers zéro quand $n$ tend vers l'infini.

Nous avons démontré que $\lim \alpha_{n}=0$.

L'ensemble $A$ et son complémentaire ayant même frontière, on obtient de la même façon $\lim \beta_{n}=0$.

Il nous reste à étudier le comportement de $\gamma_{n}$. Commençons par limiter le domaine d'intégration à $\rho \leq 2 m a_{n}$. On a

$$
0 \leq \gamma_{n}-\int_{\left[0,2 m a_{n}\right] \times A} \mathbb{P}_{\rho, \vec{u}}\left[\rho_{n-a_{n}} \in I_{n}\right] \mathrm{d} \mathcal{L}_{\rho_{a_{n}}, \vec{X}_{a_{n}}}(\rho, \vec{u}) \leq \mathbb{P}\left[\rho_{a_{n}}>2 m a_{n}\right]
$$

et $\lim \mathbb{P}\left[\rho_{a_{n}}>2 m a_{n}\right]=0$, d'après le théorème 1 .

Posons

$$
t_{n}:=\frac{t \sqrt{n\left(m^{\prime}-m^{2}\right)}+m a_{n}-\rho}{\sqrt{\left(n-a_{n}\right)\left(m^{\prime}-m^{2}\right)}}
$$

et remarquons que

$$
\mathbb{P}_{\rho, \vec{u}}\left[\rho_{n-a_{n}} \in I_{n}\right]=\mathbb{P}_{\rho, \vec{u}}\left[\rho_{n-a_{n}}-\rho \leq\left(n-a_{n}\right) m+t_{n} \sqrt{\left(n-a_{n}\right)\left(m^{\prime}-m^{2}\right)}\right] .
$$

Notons $\Phi(x):=\frac{1}{\sqrt{2 \pi}} \int_{-\infty}^{x} e^{-u^{2} / 2} \mathrm{~d} u$ la fonction de répartition de la loi normale. Le théorème 2 permet d'affirmer que, uniformément en $(\rho, \vec{u})$, on a

$$
\left|\mathbb{P}_{\rho, \vec{u}}\left[\rho_{n-a_{n}} \in I_{n}\right]-\Phi\left(t_{n}\right)\right| \longrightarrow 0 \quad \text { quand } n \rightarrow \infty .
$$

On en déduit que

$$
\gamma_{n}-\int_{\left[0,2 m a_{n}\right] \times A} \Phi\left(t_{n}\right) \mathrm{d} \mathcal{L}_{\rho_{a_{n}}, \vec{X}_{a_{n}}}(\rho, \vec{u}) \longrightarrow 0 \quad \text { quand } n \rightarrow \infty .
$$

On remarque de plus que la suite $\left(t_{n}\right)$ converge vers $t$, uniformément par rapport au paramètre $\rho$ soumis à la condition $0 \leq \rho \leq 2 m a_{n}$, et on obtient

$$
\gamma_{n}-\Phi(t) \cdot \mathbb{P}\left[\rho_{a_{n}} \leq 2 m a_{n} \text { et } \vec{X}_{a_{n}} \in A\right] \longrightarrow 0 \text { quand } n \rightarrow \infty .
$$

En réutilisant la loi des grands nombres pour le module (théorème 1) on peut conclure que

$$
\lim \gamma_{n}=\Phi(t) \cdot \lim \mathbb{P}\left[\vec{X}_{a_{n}} \in A\right]=\Phi(t) \cdot \mathbb{P}\left[\vec{X}_{\infty} \in A\right] .
$$

C'est ce que nous voulions démontrer.

\section{Questions}

Notre étude laisse de nombreuses questions ouvertes. Parmi celles-ci figurent l'étude de la loi limite de la direction et la question du théorème limite local. 
5.1. Loi limite de la direction. Nous savons que la loi limite de la direction de la marche centrifuge est supportée par la sphère toute entière, mais nous ignorons en général si elle est absolument continue par rapport à la mesure de Lebesgue sur la sphère et même si elle est continue.

Si la loi $\mu$ est invariante par les isométries vectorielles de l'espace $\mathbb{R}^{d}$ et si la marche centrifuge est issue de l'origine, alors la loi limite de la direction est uniforme sur la sphère. C'est le seul cas qui soit parfaitement clair.

Les calculs numériques effectués sur la marche centrifuge aux quatre plus proches voisins dans le plan semblent indiquer que la loi limite est continue, mais que, même si la marche est issue de l'origine, cette loi limite n'est pas uniforme sur la sphère. La figure ci-dessous représente la distribution de la variable aléatoire $\vec{X}_{1200}$, exprimée en degrés, pour la marche centrifuge plane aux quatre plus proches voisins issue de l'origine. La distribution a été représentée par un histogramme dont le pas est de 5 degrés.

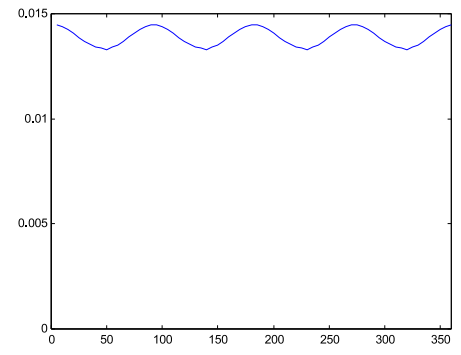

Question 1. La loi limite de la direction de la marche centrifuge est-elle toujours continue? absolument continue? Sinon, quelles sont les conditions sur la mesure $\mu$ et sur la fonction a qui l'assurent?

Puisque la marche centrifuge privilégie les directions dans lesquelles elle est déjà partie, il est clair que la loi limite de la direction dépend de la distribution initiale. La figure ci-dessous représente la distribution de la variable aléatoire $\vec{X}_{1200}$, exprimée en degrés, pour la marche centrifuge plane aux quatre plus proches voisins issue du point $(0,1)$. La distribution a été représentée par un histogramme dont le pas est de 3 degrés.

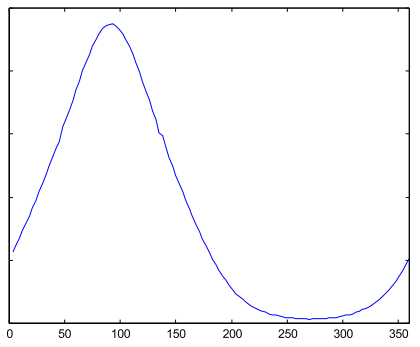

La figure ci-dessous représente la distribution de la variable aléatoire $\vec{X}_{1200}$, exprimée en degrés, pour la marche centrifuge plane aux quatre plus proches voisins issue du point $(1,1)$. La distribution a été représentée par un histogramme dont le 
pas est de 3 degrés.

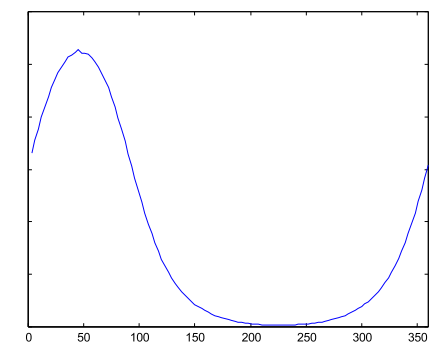

Question 2. Comment la loi limite de la direction dépend-elle de la distribution initiale de la marche centrifuge?

5.2. Théorème limite local. Une question qui nous semble particulièrement intéressante du point de vue mathématique et du point de vue de l'interprétation physique du modèle est celle du théorème limite local (TLL). Il s'agit de trouver un équivalent lorsque $n$ tend vers l'infini de la probabilité que la marche centrifuge à l'instant $n$ se trouve dans une partie bornée $K$ de l'espace. Il y a au moins deux formes possibles pour cette estimation : on peut rechercher un équivalent qui nous renseigne sur la probabilité de présence dans un ensemble $K$ fixé une fois pour toute ou bien dans un ensemble $K$ qui "part à l'infini" avec le temps. Ces deux formes sont classiques pour les marches aléatoires ordinaires et elles coïncident pour les marches centrées. La première forme du TLL est une forme précise d'estimation de grandes déviations ; elle apparaît par exemple dans un article de Stone [7] ; on l'obtient par l'analyse de Fourier dans le cas centré, associé au procédé de relativisation de Cramer ([4]) pour passer au cas décentré. La seconde forme est une version locale du théorème limite central due à Gnedenko (cf [5], §43), qui s'obtient directement par l'analyse de Fourier.

Soit $K$ une partie bornée du plan. Pour la première forme du TLL on attend un équivalent de $\mathbb{P}\left[X_{n} \in K\right]$ dont le terme principal soit de la forme $h^{n}$ avec $0<$ $h<1$. D'où la question suivante, première étape dans l'obtention d'un TLL sous sa première forme.

Question 3. Existe-t-il un nombre $h$ entre 0 et 1 tel que, pour toute distribution initiale de la marche centrifuge $\left(X_{n}\right)$ et pour toute partie bornée $K$ de l'espace,

$$
\lim _{n \rightarrow \infty} \frac{1}{n} \ln \left(\mathbb{P}\left[X_{n} \in K\right]\right)=h ?
$$

La proposition 2 donne une estimation de la transformée de Laplace du module de la marche à l'aide de paramètres $\lambda>0$ et $\gamma \in] 0,1[$. Un argument classique permet d'en déduire que, pour toute partie $K$ bornée, il existe $C>0$ tel que, pour tout $n \geq 0$,

$$
\mathbb{P}\left[X_{n} \in K\right] \leq C \gamma^{n}
$$

En d'autres termes, on a

$$
\limsup _{n \rightarrow \infty} \frac{1}{n} \ln \mathbb{P}\left[X_{n} \in K\right] \leq \ln (\gamma)<0 .
$$

Pour la marche centrifuge plane aux quatre plus proches voisins, des calculs analytiques peuvent être menés et nous savons démontrer que la constante $\gamma=\sqrt{\frac{2-a^{2}}{2}}$ 
convient. De plus le calcul numérique approché de la loi de $X_{n}$ pour de grandes valeurs de $n$ semble confirmer que l'on a effectivement

$$
\lim _{n \rightarrow \infty} \frac{1}{n} \ln \left(\mathbb{P}\left[X_{n} \in K\right]\right)=\sqrt{\frac{2-a^{2}}{2}} .
$$

(La méthode de calcul numérique utilisée a été validée sur des marches aléatoires ordinaires pour lesquelles le taux de décroissance exponentiel est connu.)

Passons à présent à une discussion sur la seconde forme du TLL.

Notons $\sigma:=\sqrt{m^{\prime}-m^{2}}$ l'écart type asymptotique de la norme $\rho_{n}$. Pour la deuxième forme du TLL, en partant de l'énoncé du TLC pour la norme et par analogie avec ce qui se passe dans le cas des marches aléatoires ordinaires, on peut conjecturer que, si la loi $\mu$ est continue, alors, pour tout $b>0$, on a

$$
\sqrt{n} \mathbb{P}\left[t \leq \rho_{n} \leq t+b\right] \sim \frac{b}{\sqrt{2 \pi} \sigma} \exp \frac{(t-n m)^{2}}{2 n \sigma^{2}},
$$

uniformément en $t \geq 0$, quand $n \rightarrow \infty$. Si la loi $\mu$ est portée par un réseau discret, on ne peut pas espérer un tel équivalent uniforme en $t$.

Question 4. Soient $A$ un borélien de la sphère $S^{d-1}$ tel que $\mathbb{P}\left[\vec{X}_{\infty} \in \partial A\right]=0$, et $b$ un nombre $>0$ fixés. A-t-on

$$
\sqrt{n} \mathbb{P}\left[t \leq \rho_{n} \leq t+b \text { et } \vec{X}_{n} \in A\right] \sim \frac{b}{\sqrt{2 \pi} \sigma} \exp \left(\frac{(t-n m)^{2}}{2 n \sigma^{2}}\right) \mathbb{P}\left[\vec{X}_{\infty} \in A\right],
$$

quand $t$ et $n$ tendent vers $+\infty$ ? De plus, si la loi $\mu$ est continue, cet équivalent est-il uniforme en $t \geq 0$, quand $n \rightarrow \infty$ ?

\section{Appendice : TLC pour martingales aVec Paramètre}

Voici le théorème limite central que nous avons utilisé dans la démonstration du théorème 2.

Théorème 6. Soit $A$ un ensemble. À chaque $a \in A$, on suppose associé un espace probabilisé $\left(\Omega_{a}, \mathcal{T}_{a}, \mathbb{P}_{a}\right)$, une filtration croissante $\left(\mathcal{F}_{a, n}\right)_{n \geq 0}$ de cet espace et une suite $\left(Y_{a, n}\right)_{n \geq 1}$ de variables aléatoires réelles intégrables adaptées et conditionnellement centrées (ie telles que pour tout $n \geq 1$, la variable $Y_{a, n}$ soit $\mathcal{F}_{a, n}$-mesurable et $\left.\mathbb{E}\left[Y_{a, n} \mid \mathcal{F}_{a, n-1}\right]=0\right)$.

On note $S_{a, n}:=\sum_{k=1}^{n} Y_{a, k}$ et $V_{a, n}:=\sum_{k=1}^{n} \mathbb{E}\left[Y_{a, k}^{2} \mid \mathcal{F}_{a, k-1}\right]$.

Si les suites $\left(Y_{a, n}\right)_{n \geq 1}$ sont uniformément bornées par un nombre indépendant de $a$ et si, pour tout $\epsilon>0$, on a

$$
\lim _{n \rightarrow \infty} \sup _{a \in A} \mathbb{P}_{a}\left[\left|1-\frac{1}{n} V_{a, n}\right|>\epsilon\right]=0,
$$

alors

$$
\lim _{n \rightarrow \infty} \sup _{a \in A, x \in \mathbb{R}}\left|\mathbb{P}_{a}\left[\frac{1}{\sqrt{n}} S_{a, n} \leq x\right]-\frac{1}{\sqrt{2 \pi}} \int_{-\infty}^{x} e^{-u^{2} / 2} d u\right|=0 .
$$

Nous avons énoncé ce théorème sous la forme que nous utilisons, pour des accroissements de martingales uniformément bornés et pour une normalisation en $\sqrt{n}$. Le résultat peut s'étendre au cas d'accroissements de martingales de carré 
intégrable, sous une condition de Lindeberg uniforme en $a$, et pour d'autres normalisations.

La littérature autour du théorème limite central pour martingales est très abondante, comme le remarque Richard Durrett dans la section 7.7 de son livre [3]. Nous donnons ici une démonstration de notre théorème en partant d'un lemme apparaissant dans des notes de cours d'Albert Raugi et Jean-Pierre Conze. La démonstration de ce lemme suit les arguments développés par Brown dans [2] pour aboutir à sa majoration (20).

Lemme 6. Soient $\left(\mathcal{F}_{k}\right)_{0 \leq k \leq n}$ une famille croissante de tribus et $\left(U_{k}\right)_{1 \leq k \leq n}$ des variables aléatoires telles que, pour $1 \leq k \leq n, \mathbb{E}\left[U_{k}^{2}\right]<\infty, U_{k}$ est $\mathcal{F}_{k}$-mesurable et $\mathbb{E}\left[U_{k} \mid \mathcal{F}_{k-1}\right]=0$.

Posons $T_{k}:=U_{1}+U_{2}+\ldots+U_{k}$ et $W_{k}:=\sum_{j=1}^{k} \mathbb{E}\left[U_{j}^{2} \mid \mathcal{F}_{j-1}\right]$. Nous supposons qu'il existe un nombre $C>0$ tel que $\mathbb{P}\left[W_{n} \leq C\right]=1$.

Alors, pour tout $\epsilon>0$, nous avons

$$
\left|\mathbb{E}\left[e^{i T_{n}+\frac{1}{2} W_{n}}\right]-1\right| \leq e^{\frac{C}{2}}\left(\left(\frac{\epsilon}{6}+\frac{\epsilon^{2}}{8}\right) C+\left(1+\frac{C}{8}\right) \sum_{k=1}^{n} \mathbb{E}\left[U_{k}^{2} \mathbf{1}_{\left|U_{k}\right|>\epsilon}\right]\right) .
$$

Nous utiliserons également une version uniforme du théorème de convergence dominée, dont la démonstration ne pose pas de difficulté, et dont voici un énoncé.

Lemme 7. Soit $A$ un ensemble. À chaque $a \in A$, on suppose associé un espace probabilisé $\left(\Omega_{a}, \mathcal{T}_{a}, \mathbb{P}_{a}\right)$ et une suite $\left(Z_{a, n}\right)_{n \geq 1}$ de variables aléatoires positives définies sur cet espace. S'il existe une constante $c>0$ telle que, pour tout $a \in A$ et tout $n \geq 1$, on ait

$$
Z_{a, n} \leq c \quad \mathbb{P}_{a}-p p
$$

et si, pour tout $\epsilon>0$,

$$
\lim _{n \rightarrow \infty} \sup _{a \in A} \mathbb{P}_{a}\left[Z_{a, n}>\epsilon\right]=0
$$

alors

$$
\lim _{n \rightarrow \infty} \sup _{a \in A} \mathbb{E}\left[Z_{a, n}\right]=0 .
$$

Preuve du Théorème. Notons $b$ une borne uniforme pour les $Y_{a, n}^{2}$. Fixons un nombre réel non nul $t$, un entier $n>0$, et notons $U_{a, k}:=\frac{t}{\sqrt{n}} Y_{a, k}$.

On a $\sum_{j=1}^{n} \mathbb{E}\left[U_{a, j}^{2} \mid \mathcal{F}_{a, j-1}\right] \leq b t^{2}$. Le lemme 6 , appliqué à la famille $\left(U_{a, k}\right)_{1 \leq k \leq n}$, nous donne :

pour tout $\epsilon>0$, et tout $n$ tel que $\epsilon \sqrt{n}>\sqrt{b}|t|$

$$
\left|\mathbb{E}\left[\exp \left(i \frac{t}{\sqrt{n}} S_{a, n}+\frac{t^{2}}{2 n} V_{a, n}\right)\right]-1\right| \leq\left(\frac{\epsilon}{6}+\frac{\epsilon^{2}}{8}\right) b t^{2} \exp \left(\frac{b t^{2}}{2}\right) .
$$

Ainsi, on a

$$
\lim _{n \rightarrow \infty} \sup _{a \in A}\left|\mathbb{E}\left[\exp \left(i \frac{t}{\sqrt{n}} S_{a, n}\right) \cdot \exp \left(\frac{t^{2}}{2 n} V_{a, n}\right)\right]-1\right|=0 .
$$

D'autre part, on a

$$
\exp \left(\frac{t^{2}}{2 n} V_{a, n}\right) \leq \exp \left(\frac{b t^{2}}{2}\right) \quad \text { pour tout } n \geq 1 \text { et tout } a \in A .
$$


L'hypothèse (27) nous permet alors d'appliquer le lemme 7 aux variables aléatoires

$$
Z_{a, n}:=\left|\exp \left(\frac{t^{2}}{2 n} V_{a, n}\right)-\exp \left(\frac{t^{2}}{2}\right)\right|
$$

et on obtient

$$
\lim _{n \rightarrow \infty} \sup _{a \in A} \mathbb{E}\left[\left|\exp \left(\frac{t^{2}}{2 n} V_{a, n}\right)-\exp \left(\frac{t^{2}}{2}\right)\right|\right]=0 .
$$

On en déduit que

$$
\lim _{n \rightarrow \infty} \sup _{a \in A}\left|\mathbb{E}\left[\exp \left(i \frac{t}{\sqrt{n}} S_{a, n}\right)\left(\exp \left(\frac{t^{2}}{2 n} V_{a, n}\right)-\exp \left(\frac{t^{2}}{2}\right)\right)\right]\right|=0 .
$$

En utilisant (28), on arrive à

$$
\lim _{n \rightarrow \infty} \sup _{a \in A}\left|\mathbb{E}\left[\exp \left(i \frac{t}{\sqrt{n}} S_{a, n}\right) \cdot \exp \left(\frac{t^{2}}{2}\right)-1\right]\right|=0 .
$$

Autrement dit la suite des fonctions caractéristiques de $\frac{1}{\sqrt{n}} S_{a, n}$ converge simplement, uniformément en $a$, vers la fonction caractéristique de la loi normale réduite.

Soit à présent une suite $\left(a_{n}\right)$ dans $A$. Notons $\mu_{n}$ la loi de la variable aléatoire $\frac{1}{\sqrt{n}} S_{a_{n}, n}$. La suite des fonctions caractéristiques des $\mu_{n}$ converge simplement vers la fonction caractéristique de la loi normale réduite. Cela garantit la convergence étroite de la suite $\left(\mu_{n}\right)$ vers cette loi normale. Et la suite des fonctions de répartition des $\mu_{n}$ converge uniformément vers la fonction de répartition $\Phi$ de la loi normale réduite.

Ainsi, on a

$$
\lim _{n \rightarrow \infty} \sup _{x \in \mathbb{R}}\left|\mathbb{P}_{a_{n}}\left(\frac{1}{\sqrt{n}} S_{a_{n}, n} \leq x\right)-\frac{1}{\sqrt{2 \pi}} \int_{-\infty}^{x} e^{-u^{2} / 2} \mathrm{~d} u\right|=0 .
$$

La suite $\left(a_{n}\right)$ étant arbitraire, on a démontré le TLC uniforme annoncé.

\section{REFERENCES}

[1] Billingsley P. Convergence of probability measures, Wiley, 1968.

[2] Brown B.M. Martingale Central Limit Theorems, The Annals of Mathematical Statistics, 42, 59-66, 1971.

[3] Durrett R. Probability : theory and examples. Wadsworth \& Brooks, 1991. (2nde édition, Duxbury Press, 1996).

[4] Cramer H. Sur un nouveau théorème limite de la théorie des probabilités, Actualités Sci. Industr. (736), 5-23, 1938.

[5] Gnedenko B.V. The theory of probability, Chelsea, 1962.

[6] Neveu J. Bases mathématiques du calcul des probabilités, Masson, 1970.

[7] Stone C. On local and ratio limit theorems, Proc. Fifth Berkeley Sympos. Math. Statist. and Probability (Berkeley, Calif., 1965/66), Vol. II: Contributions to Probability Theory, Part 2, 217-224. Univ. California Press, Berkeley, Calif. 1967. 